Revista de Comunicación y Salud, 2020, Vol. 10, n 1, pp. 67-90

Editado por Cátedra de Comunicación y Salud

ISSN: 2173-1675

RCYS

Enviado 16/03/2020

Aprobado 02/06/2020

\title{
ANÁLISIS DEL CONTENIDO PUBLICADO EN YOUTUBE, FACEBOOK E INTERNET SOBRE VACUNAS Y ANTI VACUNAS
}

\section{Analysis of pro-vaccine and anti-vaccine content published on YouTube, Facebook and internet}

\author{
Paloma Piqueiras Conlledo ${ }^{1}$ \\ Universidad Complutense de Madrid. España \\ ppiqueiras@ucm.es \\ Giorgio De Marchis \\ Universidad Complutense de Madrid. España \\ gmarchis@ucm.es
}

Victoria Cuesta Díaz

Hospital Quirón de Madrid. España

victoria.cuesta.diaz@gmail.com

Financiación. La Universidad Complutense y Santander Universidades, con el objetivo de contribuir a la promoción y mejora de la calidad de la educación superior y de la actividad investigadora, colaboran de forma conjunta en el programa de formación de investigadores y doctores al que pertenece Paloma Piqueiras.

\section{Resumen}

En este artículo se investiga la información sobre vacunas y anti vacunas que es compartida en blogs, Facebook y YouTube mediante la monitorización de publicaciones desde el año 2015 hasta la actualidad. En el análisis se ha tenido en cuenta el origen de la fuente emisora de la información, el contenido publicado, y el impacto generado. Los resultados, obtenidos del estudio de 48 vídeos de YouTube, 207 post de 5 blogs distintos y de 7 grupos de Facebook, muestran que: a) la información anti vacuna o pro vacuna encontrada en la red no suele provenir de organismo oficiales; b) el contenido compartido gira en torno a ciertos temas recurrentes y suele estar ligado a hechos puntuales (por ejemplo, un brote de sarampión o una denuncia particular por efectos secundarios de una vacuna); c) el contenido compartido carece, mayoritariamente, de carácter científico y; d) las interacciones generadas por parte de los usuarios varían mucho dependiendo de si la información es transmitida por YouTube, un blog, o

${ }^{1}$ Autora para correspondencia: Paloma Piqueiras Conlledo. Universidad Complutense de Madrid. ppiqueiras@ucm.es 
Análisis del contenido publicado en YouTube, Facebook e internet sobre vacunas y anti vacunas

Facebook. Generalmente, tanto los blogs como YouTube generan más seguidores, interacciones, y comentarios que los grupos de Facebook.

Palabras clave: Vacunas, Comunicación de salud, Facebook, YouTube, Internet.

\begin{abstract}
This article analyses the information about vaccines and anti-vaccines that is shared on blogs, Facebook, and YouTube by monitoring publications from 2015 to the present. The origin of the source of the information, the published content, and the generated engagement have been taken into account. The results, obtained from the study of 48 YouTube videos, 207 posts from 5 different blogs and 7 Facebook groups, confirm that: a) the anti-vaccine or pro-vaccine information does not usually come from official sources; b) the shared content orbits around certain recurring issues and it is usually linked to specific events (for example, a measles outbreak or a particular complaint for side effects of a vaccine); c) the shared content, generally, is not based on scientific evidence, and; d) the interactions generated by users vary greatly depending on whether the information is transmitted by YouTube, a blog, or Facebook. Generally, both blogs and YouTube generate more followers, interactions, and comments than Facebook groups.
\end{abstract}

Keywords: Vaccines, Health Communication, Facebook, YouTube, Internet.

\title{
Cómo citar el artículo
}

Piqueiras Conlledo, P., De Marchis, G. Cuesta Díaz, V. (2020). Análisis del contenido publicado en YouTube, Facebook e internet sobre vacunas y anti vacunas. Revista de Comunicación y Salud, 10(1), 67-90. doi: http://doi.org/10.35669/rcys.2020.10(1).67-90

\section{INTRODUCCIÓN}

Son varios los documentos que afirman que las vacunas son el logro de salud pública más importante del siglo XX (p.ej., World Health Organization, UNICEF, World Bank, 2009; Bean, 2011; Dube, Vivion y MacDonald, 2015, Deloitte, 2017). En datos, la Organización Mundial de la Salud (2009) ha calculado que las vacunas salvan al año alrededor de 3 millones de vidas, el equivalente a la población de Madrid o Roma. En términos de efectividad, destaca el hecho de que la vacunación ha conseguido controlar gran parte de las enfermedades existentes (Ehreth, 2003; World Health Organization, 2008) y que, incluso, ha permitido que se erradique la viruela, una patología que "una vez mató al 30\% de sus víctimas" (Bean, 2011, p. 1875) o, en América, también la poliomielitis (Dube et al., 2015).

La alta tasa de cobertura de vacunación en la mayoría de los países indica que la vacunación sigue siendo una medida de salud pública ampliamente aceptada. Así, "hoy en día se está llegando con la inmunización a más niños que en ningún otro momento de la historia" (World Health Organization, et al., 2009, p. XX) y los beneficios de las 
Análisis del contenido publicado en YouTube, Facebook e internet sobre vacunas y anti vacunas

vacunas "se están extendiendo cada vez más a los adolescentes y los adultos, protegiéndolos contra enfermedades que ponen en peligro la vida" (World Health Organization, et al., 2009, p. XX). Sin embargo, a pesar de estos datos, aún sigue existiendo cierta desconfianza en su aplicación.

Tradicionalmente, la reducción de la prevalencia e incidencia de enfermedades prevenibles por vacunación ha dependido del contexto, la política, la ciencia, la salud pública y los medios de comunicación (Dube et al., 2015, p. 99). Los facultativos también han tenido un gran protagonismo en las decisiones de los ciudadanos sobre la inmunización. En este sentido, probablemente el ejemplo más difundido sea el de Andrew Wakefield, un médico británico que en 1998 publicó en la prestigiosa revista The Lancet una investigación en la que asociaba la vacuna triple vírica con el autismo. Aunque el estudio acabó siendo un fraude, el nivel de histeria que provocaron sus afirmaciones hizo que muchos padres decidieran no vacunar a sus hijos ante el temor a que desarrollaran autismo. El resultado fue un repunte de los casos de sarampión.

Recientemente, las fuentes de referencia de información han cambiado y, por tanto, en los algoritmos de decisión de los ciudadanos sobre si vacunarse o no, han aparecido otras variables: "aunque los proveedores de atención médica son la fuente principal de asesoramiento médico, incluido el asesoramiento sobre vacunación, el público recurre cada vez más a Internet" (Bean, 2011, p. 1875). En este sentido, algunas investigaciones destacan la importancia que tiene internet en los flujos de opinión generados sobre las vacunas (Dannetun, Tegnell, Hermansson, Giesecke, 2005; Cuesta y Gaspar, 2014; Cambra, Díaz, Herrero, 2016).

Varios autores sostienen la idea de que Internet ha brindado a los anti vacunas grandes oportunidades para su exposición y que juega un papel determinante a la hora de alimentar el sentimiento anti vacunación (Davies, Chapman y Leask, 2002; Cambra, Díaz, Herrero, 2016). Siguiendo esta lógica, el nacimiento de las Redes Sociales y de Internet ha multiplicado la posibilidad de encontrar contenido erróneo y, en consecuencia, ha propiciado la toma de decisiones sobre la salud pública siguiendo informaciones inexactas o engañosas.

Basándonos en esta creencia de que los modelos tradicionales de transmisión de la información y de persuasión del mensaje de salud están cambiando, este artículo analiza el contenido anti vacuna y pro vacuna que es transmitido a través de blogs, Facebook y YouTube. Así, se pretende identificar las fuentes de información y el tipo de contenido compartido entre la comunidad de usuarios.

\section{OBJETIVOS}

El objetivo general de esta investigación consiste en analizar el tipo de información relacionada con la vacunación que se distribuye en blogs, YouTube, y grupos de Facebook.

Los objetivos específicos planteados tratan de:

Revista de Comunicación y Salud, 2020, Vol. 10, nº 1, pp. 67-90 
- Identificar las fuentes de información pro y anti vacuna;

- Conocer el contenido que publican y los temas sobre los que debaten en redes;

- Cuantificar las interacciones que son capaces de crear tanto los anti vacunas como los pro vacunas.

- Encontrar patrones de comportamiento en los generadores de contenido anti vacunas y pro vacunas en función del canal mediante el cual se distribuyen los contenidos (blogs, Facebook, YouTube).

Para alcanzar estos objetivos se plantean (Tabla 1 ) las siguientes preguntas de investigación $(\mathrm{PI})$ y sus hipótesis asociadas $(\mathrm{H})$ :

Tabla 1. Objetivos, Preguntas de investigación (PI) e Hipótesis $(H)$

\begin{tabular}{|c|c|c|}
\hline Objetivo y planteamiento & $\begin{array}{l}\begin{array}{l}\text { Preguntas } \\
\text { Investigación }\end{array} \\
\end{array}$ & Hipótesis asociada \\
\hline $\begin{array}{l}\text { Identificar a las fuentes de información pro y anti vacuna: } \\
\text { en internet el contenido generado no suele pasar filtros } \\
\text { de veracidad y su control no es exhaustivo, por lo que se } \\
\text { hace imprescindible conocer las fuentes de la } \\
\text { información publicada. }\end{array}$ & $\begin{array}{lr}\text { PI1: ¿Quiénes } & \text { son } \\
\text { las fuentes de } & \text { del } \\
\text { emisión } & \text { del } \\
\text { contenido } & \text { pro } \\
\text { vacunas y anti } \\
\text { vacunas en blogs, } \\
\text { Facebook ry } \\
\text { YouTube? }\end{array}$ & $\begin{array}{l}\text { H1: Las fuentes de } \\
\text { información transmitidas } \\
\text { en internet no son } \\
\text { organismos oficiales. }\end{array}$ \\
\hline $\begin{array}{l}\text { Conocer el contenido que publican y los temas sobre los } \\
\text { que debaten en redes: la información que se propaga por } \\
\text { internet es de fácil acceso para la mayoría de la } \\
\text { población, lo que supone que cualquier persona es } \\
\text { susceptible de ser influenciada por los contenidos } \\
\text { compartidos en YouTube, blogs o Facebook. Por esta } \\
\text { capacidad de influencia, en esta investigación se plantea } \\
\text { como imprescindible conocer y entender el contenido } \\
\text { que se genera en los canales objeto de estudio. }\end{array}$ & $\begin{array}{l}\text { PI2: ¿Cuál es el } \\
\text { contenido } \\
\text { transmitido? }\end{array}$ & $\begin{array}{l}\text { H2: Los contenidos } \\
\text { responden a temas de } \\
\text { actualidad. }\end{array}$ \\
\hline $\begin{array}{l}\text { Cuantificar las interacciones que son capaces de crear } \\
\text { tanto los anti vacunas y pro vacunas: recalcando la } \\
\text { capacidad de influencia que tienen las Redes Sociales e } \\
\text { internet, en esta investigación se cuestiona cuánto } \\
\text { engagement generan las posturas enfrentadas sobre la } \\
\text { vacunación. }\end{array}$ & $\begin{array}{lr}\text { PI3: } & \text { ¿Quién } \\
\text { consigue } & \text { más } \\
\text { interacciones: } & \text { los } \\
\text { emisores } & \text { de } \\
\text { contenidos } & \text { anti } \\
\text { vacunas o los de } \\
\text { contenido } \\
\text { vacunas? }\end{array}$ & $\begin{array}{l}\text { H3: Los contenidos a } \\
\text { favor de la vacunación } \\
\text { generan más capacidad } \\
\text { de influencia que los } \\
\text { contenidos anti } \\
\text { vacunas. }\end{array}$ \\
\hline $\begin{array}{l}\text { Encontrar patrones de comportamiento en los } \\
\text { generadores de contenido anti vacunas y provacunas en } \\
\text { función del canal mediante el cual se distribuyen los } \\
\text { contenidos: dado que los tres canales analizados ofrecen } \\
\text { posibilidades distintas tanto a autores como a usuarios, } \\
\text { nos planteamos si existen unos medios más propicios } \\
\text { que otros a la hora de crear contenido pro o anti } \\
\text { vacunación. }\end{array}$ & $\begin{array}{lr}\text { PI4: ¿En qué } \\
\text { medios son capaces } \\
\text { de generar } & \text { más } \\
\text { interacciones r los } \\
\text { contenidos yro } \\
\text { vacunas y anti } \\
\text { vacunas? }\end{array}$ & $\begin{array}{l}\text { H4: Facebook y } \\
\text { YouTube atraerán más } \\
\text { contenido anti vacunas, } \\
\text { mientras que en los } \\
\text { blogs se tenderá a } \\
\text { compartir información } \\
\text { pro vacunación. }\end{array}$ \\
\hline
\end{tabular}

Fuente: Elaboración propia 


\section{METODOLOGÍA}

En el presente artículo se detallan los datos recopilados sobre vacunas y anti vacunas registrados en distintos blogs, canales de YouTube, y grupos de Facebook. Los datos que aparecen en este documento fueron recopilados entre el 23 de diciembre de 2019 y el 23 de enero de 2020.

La metodología ha sido desarrollada en dos pasos: a) por un lado, se estipuló un procedimiento exhaustivo de registro de los datos y; b) por otro, se definieron las especificidades de cada campo de búsqueda.

El esquema general de recolección de datos es el mismo para todos los canales analizados (origen de la fuente, contenido e interacciones). Sin embargo, la información registrada varía en función de las posibilidades de investigación de cada medio (YouTube, Facebook, y blogs). En consecuencia, se ha tenido en cuenta que en YouTube las interacciones se pueden contabilizar en likes positivos y negativos, pero que en los blog no es posible hacer este registro. De la misma forma, se ha tomado en consideración la posibilidad que ofrecen algunos blogs de compartir el contenido de forma directa en otras Redes Sociales, como Twitter y LinkedIn, a pesar de que en los otros canales estudiados esa opción no es posible.

A continuación se detalla la construcción del método y, en última instancia, se describe la muestra utilizada.

\subsection{Procedimiento de registro de los datos}

Para proceder al registro de los datos se tuvieron en cuenta las siguientes especificidades de cada canal:

- YouTube

o Idioma: vídeos en español.

- Palabras clave: el título del vídeo contiene la palabra "vacuna(s)" o "anti vacuna(s)".

o Filtros: haciendo uso del buscador de la aplicación se filtró introduciendo tanto las palabras clave descritas en el punto anterior, como las fechas que se señalan en el punto siguiente.

- Periodo: la fecha de publicación del vídeo es igual o posterior al 1 de enero de 2015.

- Blogs

o Idioma: contenido en español.

- Palabras clave: la palabra "vacuna(s)" o "anti vacuna(s)" está contenida en el texto.

- Filtros: El proceso de búsqueda se hizo mediante las etiquetas de los propios blogs o filtrando a través de sus campos de búsqueda. 
Análisis del contenido publicado en YouTube, Facebook e internet sobre vacunas y anti vacunas

o Periodo: la fecha de publicación del post es igual o posterior al 1 de enero de 2015.

- Restricciones: para que un post fuera tenido en cuenta se contabilizó el número de entradas que el autor del blog había hecho sobre el contenido a analizar. De tal forma que, cualquier blog con menos de cinco textos referidos a las vacunas era descartado.

- Facebook

o Idioma: contenido en español.

- Palabras clave: el título del grupo contiene la palabra "vacuna(s)" o "anti vacuna(s)".

o Filtros: se seleccionó la opción "grupos" en la búsqueda, descartando otras posibilidades de investigación como "publicaciones", "páginas" o "vídeos".

- Periodo: los grupos tienen una actividad mínima de 10 publicaciones al año desde el 1 de enero de 2015.

o Restricciones: análisis de los grupos de acceso público. Se descartaron los grupos privados por la imposibilidad de ver el contenido compartido.

\subsection{Explicación de los campos registrados en cada canal}

De forma genérica, el análisis del contenido de los canales escogidos se estructura en tres grandes bloques:

1) El origen de la fuente para recoger los datos sobre la autoría del vídeo.

2) La descripción del vídeo para detallar el contenido y la calidad del mismo.

3) El nivel de engagement generado para dar cuenta del impacto que ha tenido el contenido en los usuarios.

De forma específica, los campos de registro dentro de cada bloque se describen a continuación:

\subsubsection{YouTube}

- Origen de la fuente.

o Nombre del autor: nombre de la persona que es autora del vídeo.

o Nombre del canal: nombre del canal donde se inserta el vídeo.

- País - continente: país de origen del vídeo o continente (en caso de que no se conozca exactamente el país).

- Descripción del contenido.

o Título: título del vídeo.

- Fecha: fecha en la que se subió el vídeo a YouTube.

o Duración: tiempo en segundos.

o Resumen: sinopsis del contenido del vídeo.

o Género: modo de transmisión de la información. Hay cinco opciones: 
- Monólogo (el autor del vídeo intercala imágenes con declaraciones con voz en off y su propia presencia);

- Noticia (el vídeo trata sobre una noticia de un informativo o programa de TV);

- Reportaje (el vídeo es un reportaje);

- Entrevista (se retransmite una entrevista periodística);

- Debate (se muestra un debate sobre las vacunas).

o Fuente principal: la persona que genera el vídeo. Hay cinco opciones posibles:

- Persona particular (se trata de un bloguero que incluye el vídeo en su canal);

- Periodista (el vídeo es un fragmento de un reportaje o de un programa de televisión donde el conductor de la charla o del contenido es un periodista);

- Colectivo de periodistas (la fuente que crea el vídeo es un colectivo y no un único periodista);

- Profesional sanitario (es un vídeo creado y difundido por un médico).

- Fuente secundaria: la fuente secundaria sólo aparece cuando el vídeo refleja una entrevista o un reportaje. Entonces se registra a qué persona se le está haciendo la entrevista o quién participa en el reportaje. Hay seis opciones posibles:

- Médico (la persona a la que el periodista pregunta -fuente principalen la entrevista o el reportaje es un médico);

- Ciudadano (se incluyen declaraciones de ciudadanos en el reportaje que estamos viendo en el vídeo);

- Médico y ciudadanos (las personas que aparecen en el reportaje donde la fuente principal es un periodista- son médicos que aportan una visión científica y ciudadanos que contribuyen con su opinión);

- Declaraciones oficiales del gobierno (durante el desarrollo de la noticia se insertan declaraciones de un miembro del gobierno);

- Experto (se realiza la entrevista a un experto en la materia pero no es un médico).

o Postura: opinión con respecto a las vacunas. Hay cuatro opciones posibles:

- Pro vacuna (a favor de la vacunación);

- Anti vacuna (en contra de la vacunación);

- Neutro (no se aprecia opinión ni a favor ni en contra);

- Ambivalente (se confrontan las dos posiciones, por lo se establece un debate entre aquellos intervinientes que se muestran a favor y quienes se posicionan en contra de las vacunas).

- Calidad: se refleja si el contenido está sustentado en fuentes científicas (contenido de calidad) o si no existen referencias claras a las fuentes de la información (contenido sin calidad).

- Nivel de interacciones generado. 
o Visualizaciones: número de visualizaciones del vídeo en el momento de la fecha de registro.

o Likes: número de likes del vídeo en el momento de la fecha de registro. Se registran tanto los positivos como los negativos.

o Suscripciones: número de suscripciones al canal.

o Comentarios: comentarios positivos, negativos y neutros realizados al vídeos por los usuarios. En los vídeos con menos de 50 comentarios se contabilizó el tanto por ciento de positivos, negativos y neutros. En los vídeos con más de 50 comentarios se seleccionó una muestra (los primeros 20 comentarios) y se extrapolaron los resultados al total. Para hacerlo, se multiplicó por 100 el número de comentarios positivos, negativos y neutros de la muestra y se dividió por el número total de comentarios escogidos

porcentaje de comentarios $=\frac{\text { comentarios neutos,positivos o negativos } x 100}{\text { número de comentarios seleccionados como muestra }}$ ).

\subsubsection{Blogs}

- Origen de la fuente.

o Nombre del autor: nombre de la persona que es autora del post.

o Nombre del blog: nombre del blog donde se inserta el post.

o Tipo de fuente: si la fuente es oficial (un organismo oficial) o no oficial (un particular o conjunto de profesionales).

- Descripción del contenido.

o Título del post.

o Resumen: sinopsis del contenido.

o Tipo de contenido: si se basa en información, en opinión del autor o en datos científicos y estadísticas. Las fórmulas pueden estar combinadas.

- Postura: Opinión con respecto a las vacunas. Hay tres opciones posibles:

- Pro vacuna (a favor de la vacunación);

- Anti vacuna (en contra de la vacunación);

- Neutro (no se aprecia opinión ni a favor ni en contra en ese post);

No se han registrado resultados de la postura ambivalente, aunque sí se ha tenido en cuenta este criterio de búsqueda.

- Fecha: el día de publicación del post.

- Calidad: se parece a la columna "tipo de contenido" pero, en este caso, se trata de distinguir si el contenido compartido está sustentado en fuentes reseñables y de calidad o no.

- Nivel de engagement generado.

o Likes: número de likes del post en el momento de la fecha de registro.

- Lecturas: número de lecturas del post hasta la fecha de registro.

o Suscripciones: número de suscripciones al blog.

o Comentarios: comentarios realizados por los usuarios. Hay 3 posibilidades:

- Positivos;

- Negativos; 
Análisis del contenido publicado en YouTube, Facebook e internet sobre vacunas y anti vacunas

- Neutros.

o Contenido compartido: Se registra cuántas veces el contenido ha sido compartido de forma directa desde el blog en otras Redes Sociales como Facebook, Linkedln y Twitter.

\subsubsection{Facebook}

- Origen de la fuente.

o Tipo de fuente: se registra el nombre del grupo en Facebook y el tipo de perfil (si es una fuente oficial o no).

- Descripción del contenido.

o Postura: Se registra si el grupo tiene contenido:

- Pro vacunas (a favor de la vacunación) o;

- Anti vacunas (en contra de la vacunación).

o Tipo de contenido: si el contenido es científico, informativo o anecdótico. Las opciones pueden estar combinadas.

o Inicio de las publicaciones: comienzo de la actividad del grupo.

o Calidad del contenido: se registra si es:

- Apropiado (si reproduce contenido sobre vacunas) o;

- Inapropiado (si el contenido compartido son chistes, imágenes, etc.)

- Nivel de engagement generado.

o Miembros: número de personas que pertenecen al grupo.

\subsection{Muestra}

La muestra de esta investigación está compuesta por 48 vídeos de YouTube, 207 entradas de 5 blogs distintos con tendencias dispares y 7 grupos de carácter público de Facebook. La información se especifica en el Anexo 1.

\section{RESULTADOS Y DISCUSIÓN}

En este apartado se describen los resultados de la investigación en función de las preguntas de investigación planteadas.

Pregunta de investigación 1 (PI): La información compartida en los medios estudiados es mayoritariamente emitida por fuentes no oficiales. En esta investigación se entiende por fuentes oficiales, organismos gubernamentales, administraciones locales o autonómicas, hospitales y profesionales de la medicina.

Específicamente, se han detectado dos vídeos en YouTube que pertenecen a especialistas médicos. En los 46 vídeos restantes las autorías se reparten entre (Gráfico 1): blogueros (12 vídeos, un 25\%); medios de comunicación (33 vídeos, un $69 \%$ ) y un colectivo de periodistas (1 vídeo, el 2\%). 


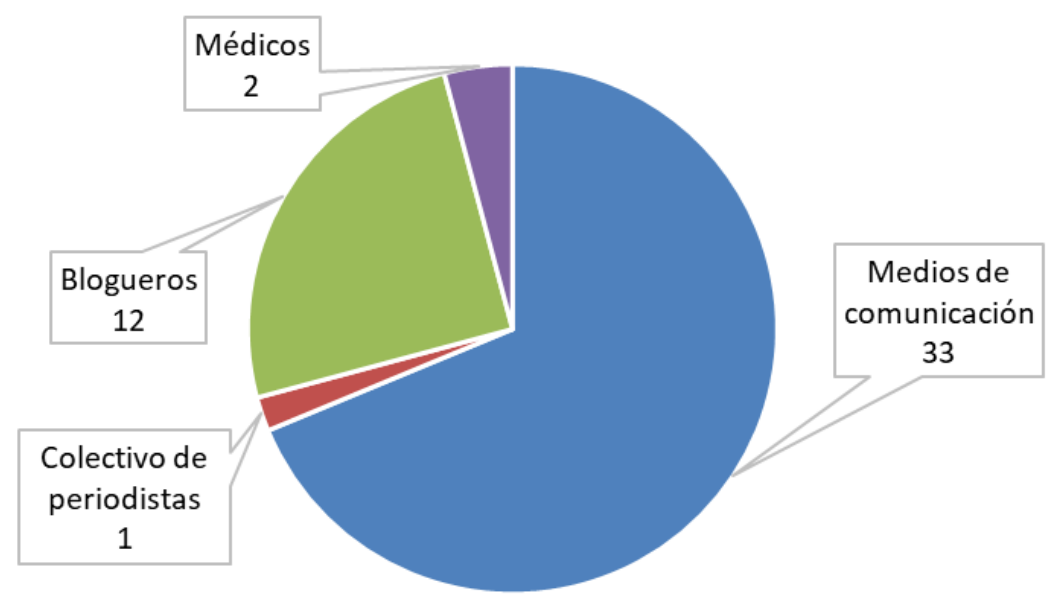

Gráfico 1. Autoría de los vídeos analizados.

Fuente: Elaboración propia.

De los grupos señalados en el Gráfico 1, se comprueba que aquellos vídeos procedentes de medios de comunicación tienen una característica especial: en el 63,3\% de los casos (21 vídeos) el medio recurre a fuentes oficiales para dotar de calidad al contenido. Así: a) en los 6 vídeos en los que se retransmite una noticia se registran declaraciones oficiales sobre el hecho narrado; b) en 5 de los 10 vídeos en los que se recogen entrevistas el periodista pregunta a expertos; c) en 5 de los 10 reportajes analizados, se incluyen declaraciones de médicos; d) en los 6 vídeos que muestran debates sobre la vacunación, tan sólo hay uno en el que no participan médicos y; e) en el único vídeo emitido por un programa de humor, el periodista realizó un monólogo sin recurrir a fuentes.

En la exploración realizada en los grupos de Facebook no se ha encontrado ningún grupo de carácter oficial.

Dentro de los blogs, se considera al Real e Ilustre Colegio de Farmacéuticos de Sevilla (RICFS) como una fuente oficial, así como al Blog de Pills y al Blog de Live Med Iberia. En el caso de los dos últimos, aunque no representan a ningún organismo oficial, sus post están escritos por miembros del colectivo sanitario (especialmente, médicos y farmacéuticos).

Basándonos en estos datos se valida la Hipótesis 1:

H1: Las fuentes de información transmitidas en internet no son organismos oficiales.

Pregunta de Investigación 2 (PI2): Los contenidos analizados responden a temas recurrentes y de actualidad, pero la forma de transmisión y el enfoque de la información varía dependiendo del medio explorado. A este respecto, existen claras diferencias entre un vídeo de YouTube, un blog o una publicación en Facebook. 
En YouTube la publicación de los vídeos está directamente ligada al contenido de actualidad. En este sentido, se pone de manifiesto que tanto los medios de comunicación como los blogueros y el colectivo de periodistas publican sus informaciones cuando la vacunación es noticia. Por el contrario, en los dos casos de vídeos publicados por médicos, el motivo de la emisión no es la actualidad, sino la transmisión de sus propias opiniones sobre los beneficios de la vacunación.

El contenido de los blogs es muy dispar y resulta dificultoso encontrar patrones comunes entre ellos. No obstante, para facilitar la exposición de los resultados se han establecido tres categorías: 1) Información recurrente: se puede establecer con claridad un índice de temas tratados. Dentro de esta categoría se registra el blog de Miguel Jara en el que la información sobre la vacuna contra el Virus del Papiloma Humano (VPH), la crítica a la industria farmacéutica o la supuesta ineficacia de la vacuna del sarampión ocupan gran parte de los post; 2) Consultorio: los autores dan respuesta en sus post a las preguntas de los usuarios. Se detecta este patrón de comportamiento en los blogs del RICFS, isalud y Live Med Iberia; 3) Miscelánea: La gran variedad de temas hace imposible su catalogación. El blog de Pills se caracteriza por publicar contenido pro vacuna con información sustentada en datos y estadísticas, pero sus publicaciones no están conectadas.

En lo referente a Facebook se han identificado 8 grupos públicos con contenido relacionado con las vacunas, pero en el estudio final sólo se han considerado 7 de ellos (Tabla 2). Esto es debido a que uno de los grupos no ofrece contenido acorde con la temática de estudio.

Tabla 2. Información sobre los grupos pro o anti vacunas en Facebook.

\begin{tabular}{|l|l|l|l|l|}
\hline Nombre del grupo & Tendencia & $\begin{array}{l}\text { Tipo de } \\
\text { contenido }\end{array}$ & $\begin{array}{l}\text { Periodicidad } \\
\text { publicación }\end{array}$ & Miembros \\
\hline STOP VACUNAS & Anti & Informativo & 40 publicaciones al día & 530 \\
\hline $\begin{array}{l}\text { No vacunas forzadas - No } \\
\text { SB277 }\end{array}$ & Anti & Informativo & 8 publicaciones al mes & 270 \\
\hline Afectados por el mercurio & Anti & Informativo & $\begin{array}{l}7 \text { publicaciones a la } \\
\text { semana }\end{array}$ & 178 \\
\hline Científicos provacunas & Pro & Informativo & 12 publicaciones al año & 256 \\
\hline $\begin{array}{l}\text { iLa importancia de las } \\
\text { Vacunas! }\end{array}$ & Pro & Informativo & $\begin{array}{l}2 \text { publicaciones a la } \\
\text { semana }\end{array}$ & 441 \\
\hline Anti Antivacunas & Pro & $\begin{array}{l}\text { Informativo- } \\
\text { anecdótico }\end{array}$ & $\begin{array}{l}6 \text { publicaciones a la } \\
\text { semana }\end{array}$ & 106 \\
\hline $\begin{array}{l}\text { El peligro del mercurio en } \\
\text { las vacunas }\end{array}$ & Anti & $\begin{array}{l}\text { Informativo- } \\
\text { anecdótico }\end{array}$ & 12 publicaciones al año & 332 \\
\hline
\end{tabular}

Fuente: Elaboración propia.

El contenido de estos grupos es muy genérico, pero está asociado a temas de actualidad. De hecho en todos los grupos se comparte contenido mediático. De esta 
Análisis del contenido publicado en YouTube, Facebook e internet sobre vacunas y anti vacunas

forma, un usuario publica una noticia de un medio y la comunidad de Facebook interesada hace comentarios e interactúa con ella.

La información aportada valida la segunda Hipótesis:

H2: Los contenidos responden a temas de actualidad.

Pregunta de investigación 3 (PI3): Esta pregunta de investigación no ha podido ser contestada, debido a que la forma de contabilizar las interacciones o la capacidad de influencia no es comparable entre YouTube, Facebook, y blogs.

Es posible afirmar que las interacciones en los vídeos de YouTube son significativas (aproximadamente la mitad de los vídeos analizados, 20 de los 48, tienen más de 100 comentarios) y que las valoraciones realizadas a los contenidos de los grupos de Facebook son muy escasas (apenas hay publicaciones que superen los 5 "me gusta"). Sin embargo, como los métodos de medición son distintos, no podemos establecer relaciones directas ni concluir qué medio tiene más seguidores.

No obstante, como parte de los resultados, se aportan varias comparaciones en función de los medios analizados:

En YouTube es destacable el nivel de interacciones generadas. Por autores: a) los vídeos procedentes de medios de comunicación superan las 606.000 visualizados y generan 19.223 likes positivos y 1.047 negativos; b) el total de visualizaciones alcanzadas por los vídeos analizados que son emitidos por blogueros alcanzan la cifra de 2.212.313. Asimismo, el total de likes positivos es de 170.383, mientras que los negativos apenas superan los 4.000. Además, hay 21.005 comentarios registrados en estas publicaciones de los cuales el $38 \%$ se muestra a favor de las vacunas, el $41 \%$ mantiene una postura neutral y el $21 \%$ se declara anti vacunas (Gráfico 2); c) por último, los dos vídeos realizados por profesionales médicos suman 133.887 visualizaciones y tienen la peculiaridad de obtener más likes negativos (134.211) que positivos (991).

Revista de Comunicación y Salud, 2020, Vol. 10, nº 1, pp. 67-90 


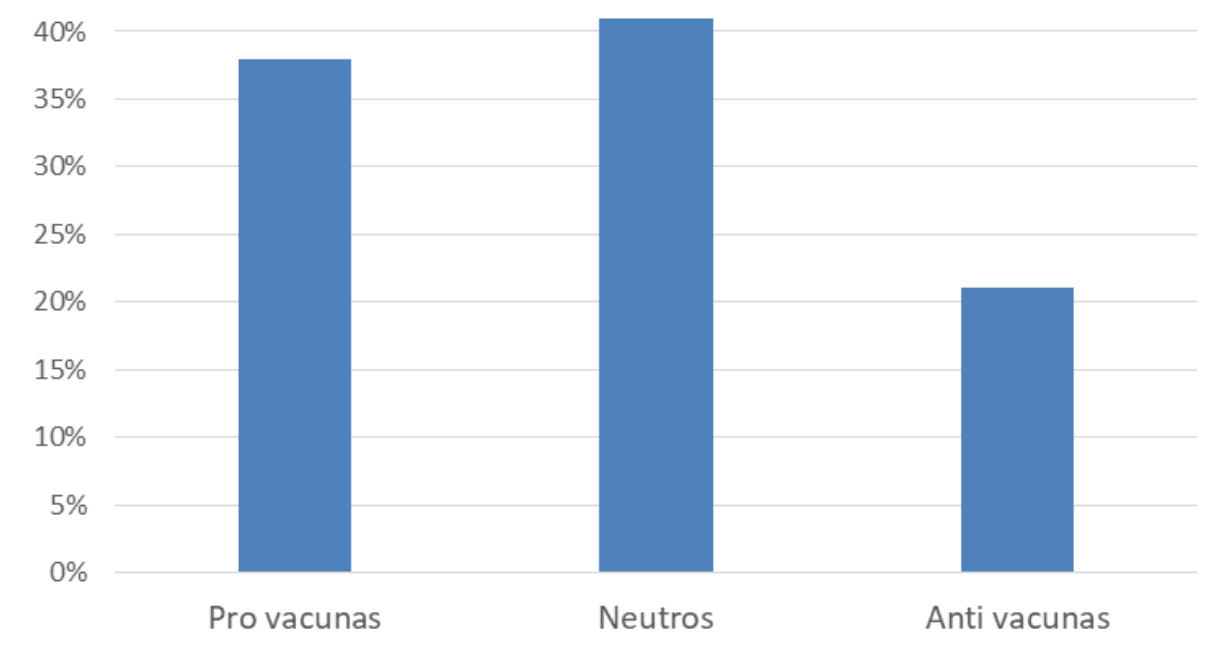

Gráfico 2. Tendencias de los comentarios hechos a los vídeos de blogueros.

Fuente: Elaboración propia.

En el caso de los blogs resulta complicado poder hacer valoraciones. Los datos recopilados son muy dispares debido a que las variables de medición no coinciden en todas las publicaciones. Esto supone que del Blog de Pills sólo se hayan podido contabilizar los comentarios (29 en total) y del blog de Live Med Iberia tan sólo se hayan podido registrar las tasas de lectura de cada entrada (una media de 1.060 por publicación). Por su parte, en el blog del RICFS y en el de isalud no se especifica ninguna de las variables utilizadas para medir las interacciones. Por este motivo, a continuación, sólo se exponen con detalle los datos del Blog de Miguel Jara.

Aunque su número de suscriptores no es público, se ha contabilizado que Jara acumula 575.858 likes en las publicaciones analizadas (una media de 4.500 por publicación) y su contenido es masivamente compartido por sus lectores (Gráfico 3) en Facebook (972.066 veces), Twitter (14.501) y Linkedln (632). Además, el total de comentarios en sus entradas es de 1.100, una cifra muy superior a los comentarios hechos, por ejemplo, en el Blog de Pills (29). 


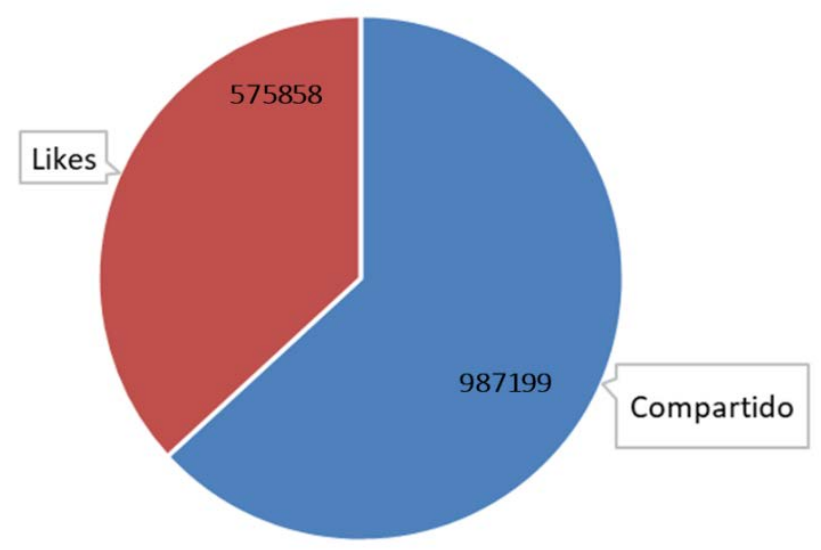

Gráfico 3. Interacciones del blog de Miguel Jara contabilizadas en "likes" y en contenido compartido en otras Redes Sociales.

Fuente: Elaboración propia.

Las interacciones en Facebook son las menos representativas de toda la muestra. Al comparar con los otros medios analizados, comprobamos que aunque los grupos estudiados suelen tener más de un centenar de seguidores, su actividad e interacciones son muy reducidas.

La periodicidad de publicación de contenidos varía en función del grupo que se estudie pero, de forma genérica, los contenidos son compartidos semanal y mensualmente. Por su parte, las reacciones a esos contenidos compartidos son muy escasas (una publicación media tiene 3 "me gusta", prácticamente no suscita comentarios, y su índice de impacto es menor, en casi todos los casos, a las 50 visualizaciones).

Pregunta de investigación 4 (PI4): Se presentan los resultados de la PI4 sobre la capacidad que tienen los medios estudiados para generar interacciones pro o anti vacunas.

En la exploración de los datos se ha observado que tanto en los vídeos de YouTube como en los blogs analizados la tendencia es pro vacunación: el 60,4\% de los vídeos y el $80 \%$ de los blogs son favorables a la inmunización. Sin embargo, menos de la mitad de los grupos de Facebook $(42,8 \%)$ se muestran a favor de las vacunas.

A priori, se detectó que los contenidos anti vacunas encontraban en Facebook un medio más afable para compartir sus informaciones. Sin embargo, conviene matizar esta afirmación añadiendo una apreciación importante con respecto a los blogs: el blog de Miguel Jara, único blog detectado con tendencia contraria a la vacunación, tiene el doble de capacidad para generar contenido e interacciones que los otros 4 blogs juntos (Tabla 3). Así, de las 207 entradas analizadas en la categoría, 128 pertenecen a Jara. 
Análisis del contenido publicado en YouTube, Facebook e internet sobre vacunas y anti vacunas

Tabla 3. Comparativa de las interacciones en blogs.

\begin{tabular}{|c|c|c|c|c|c|c|}
\hline Blog & $\begin{array}{l}\mathrm{N}^{\circ} \text { de } \\
\text { post } \\
\text { analiz. }\end{array}$ & Suscript. & $\begin{array}{l}\text { Media de likes } \\
\text { por public. }\end{array}$ & $\begin{array}{l}\text { Contenido } \\
\text { compartido }\end{array}$ & $\begin{array}{l}\text { Coment. } \\
\text { totales }\end{array}$ & $\begin{array}{l}\text { Media de } \\
\text { lecturas }\end{array}$ \\
\hline \multirow{3}{*}{$\begin{array}{l}\text { Miguel } \\
\text { Jara }\end{array}$} & \multirow{3}{*}{128} & \multirow{3}{*}{ Desconocido } & \multirow{3}{*}{4.500} & $\begin{array}{c}\text { Facebook: } \\
972.066\end{array}$ & \multirow{3}{*}{1.100} & \multirow{3}{*}{ Desconocido } \\
\hline & & & & Twitter: 14.501 & & \\
\hline & & & & LinkendIn: 632 & & \\
\hline $\begin{array}{l}\text { El blog de } \\
\text { PIlls }\end{array}$ & 9 & Desconocido & Desconocido & Desconocido & 29 & Desconocido \\
\hline $\begin{array}{l}\text { Live Med } \\
\text { lberia }\end{array}$ & 52 & Desconocido & Desconocido & Desconocido & Descon. & 1.060 \\
\hline RICFS & 12 & Desconocido & Desconocido & Desconocido & Descon. & Desconocido \\
\hline isalud & 6 & Desconocido & Desconocido & Desconocido & Descon. & Desconocido \\
\hline
\end{tabular}

Fuente: Elaboración propia.

Los datos expuestos en el análisis permiten refutar la Hipótesis 4:

H4: Facebook y YouTube atraerán más contenido anti vacunas, mientras que en los blogs se tenderá a compartir información pro vacunación.

Durante la investigación se ha detectado que el contenido anti vacunas se comparte más en Facebook y en blogs. Por el contrario, el contenido favorable a la vacunación se localiza mayoritariamente en YouTube.

\section{CONCLUSIONES}

Retomando el esquema de la metodología, las conclusiones que se detallan a continuación se estructuran en: 1) origen de la fuente, 2) contenido de la información, y 3) interacciones generadas.

\subsection{Según el origen de la fuente}

- La información anti vacuna o pro vacuna encontrada y analizada no surge, en la mayoría de los casos, de fuentes oficiales.

No se han detectado grupos de Facebook con autoría gubernamental o administrativa. En el caso de Facebook sólo 2 de los 48 vídeos proceden de fuentes médicas y, en el caso de los blogs el único que representa a una institución de profesionales colegiados es el Blog del Colegio de Farmacéuticos, en cuanto a que representa a una institución de profesionales colegiados.

- Las fuentes de información suelen ser colectivas en el caso de los Blogs, pero individuales en Facebook y YouTube. 
Lo más habitual en Facebook y YouTube es encontrar a personas particulares que dan su opinión sobre la vacunación. Si bien es cierto que la mayoría utiliza un seudónimo para publicar sus contenidos, cada una de las personas que participa es fácilmente identificable en la medida en que sus nombres están ligados a un perfil dentro de la propia Red Social.

Esto no sucede en los Blogs: 4 de los 5 analizados están gestionados por un colectivo (RICFS) o por un compendio de profesionales (El blog de Pills, Live Med Iberia, El blog de isalud). No obstante, tan sólo en los casos del RICFS y en Live Med Iberia conocemos el nombre del autor de la información, mientras que, en el resto, la entrada la firma un colectivo (por ejemplo, "equipo de isalud").

Cabe resaltar que el caso del blog de Miguel Jara es diferente. El autor es perfectamente identificable y se hace responsable de la información que él comparte dentro de su espacio.

\subsection{Según el contenido}

- El contenido varía en función del medio analizado.

Se ha identificado que los temas compartidos en Youtube responden a la coyuntura del momento. Esto supone que el patrón de publicaciones que se sigue en este medio está ligado a la actualidad.

Con respecto a los blogs se han registrado distintas categorías para estructurar el contenido: a) Información recurrente; b) Consultorio, y c) Miscelánea). Sin embargo, no se han encontrado similitudes claras en las informaciones compartidas en cada blog.

Finalmente, en el estudio de los grupos de Facebook se puede concluir que la información se publica en función de los temas candentes de actualidad sobre vacunación.

- El contenido publicado suele mostrarse a favor de la vacunación o en contra de la misma en función del medio por el que se transmita la información.

Se aprecia una cierta tendencia de apoyo de la vacunación en YouTube y, en cierto sentido, también es palpable un rechazo a los contenidos que son contrarios a la misma. No obstante, en los vídeos compartidos por medios de comunicación es habitual ver cómo se cede espacio a las personas declaradas anti vacunas ofreciéndoles un protagonismo equiparable al de los pro vacunas.

El contenido anti vacunas es más habitual en blogs (128 entradas responden a contenido anti vacunas frente a 79 entradas a favor) y en Facebook (4 de los 7 grupos comparten contenido contrario a la vacunación). 
- La forma de transmisión de la información varía según el canal estudiado.

Mientas que en YouTube se cede protagonismo a los debates y a las entrevistas, en Facebook y en los blogs lo habitual es que un sujeto transmita información u opinión de manera unidireccional.

\subsection{Según las interacciones generadas}

- Las interacciones en Facebook son mucho menores que las generadas en YouTube y en los blogs.

El nivel de comentarios y de "likes" conseguidos en YouTube es mucho más contundente que en el resto de canales, quizás porque los usuarios son capaces de interactuar con el contenido de forma más sencilla.

No obstante, a través de un blog también es posible generar interacciones. El paradigma es Miguel Jara: acumula una gran cantidad de comentarios y tiene la capacidad de hacer que su contenido sea reproducido por los usuarios en otras Redes Sociales como son Linkedln o Twitter. Sin embargo, esto no sucede con los otros 4 blogs analizados, cuyo nivel de interacciones es ínfimo.

Por su parte, en Facebook las interacciones son muy limitadas.

- No se puede determinar que las interacciones en los blogs sean reales.

Mientras que en Facebook y Youtube los autores de los comentarios pueden ser identificados, en los blogs no sucede lo mismo. En este medio, el nombre de las personas que comparten el contenido no es público y el acceso a las mismas a través de los comentarios no es posible. Esto dificulta la identificación de DOLs (Digital Opinion Liders) y pone en duda si el autor tiene tantos seguidores como se deduce de las interacciones.

- Las interacciones generadas hacia el contenido anti vacunas son más representativas en los blogs.

Esta conclusión puede ser comprobada analizando los 5 blogs propuestos en esta investigación. De ellos, tan sólo 1 es anti vacunas y su nivel de seguidores, comentarios e interacciones es llamativamente superior a los otro 4. También es cierto que el contenido generado por Miguel Jara es muy superior al que produce el resto de blogs.

En el caso de YouTube, se detecta la tendencia contraria: la cantidad de comentarios y de "likes" aumenta si el contenido compartido es pro vacuna. Así, los dos únicos vídeos registrados como anti vacunación acumulan en total 11 comentarios, 1197 visualizaciones, 37 "likes" positivos y 3 "likes" negativos. Son números irrisorios si se tiene en cuenta que entre los dos canales tienen más de 340.000 suscriptores. 
Análisis del contenido publicado en YouTube, Facebook e internet sobre vacunas y anti vacunas

En el análisis de los grupos de Facebook no se puede llegar a ninguna conclusión sobre las interacciones realizadas, ya que, aunque el número de miembros es superior en los grupos anti vacunas (1.000 frente a 352), el nivel de interacción es bajo en todos ellos.

\section{REFERENCIAS}

Bean, S. J. (2011). Emerging and continuing trends in vaccine opposition website content. Vaccine, 29(10), 1874-1880. doi: https://doi.org/10.1016/j.vaccine.2011.01.003

Cambra, U. C., Díaz, V. C., \& Herrero, S. G. (2016). Vacunas y anti vacunas en la red social Youtube. Revista de Ciencias Humanas y Sociales, (9), 447-465. Disponible en: https://dialnet.unirioja.es/servlet/articulo?codigo=5891177

Cuesta, Ubaldo y Gaspar, Sandra. (2014). La "reputación online" de la información de vacunas en internet". Historia y comunicación social. Vol.19, 15-29. doi: https://doi.org/10.5209/rev HICS.2014.v19.45007

Dannetun, E., Tegnell, A., Hermansson, G., and Giesecke, J. (2005A telephone survey. Scand J Prim Health Care 23(3), 149-53. doi: https://doi.org/10.1080/02813430510031306

Davies, P., Chapman, S., \& Leask, J. (2002). Antivaccination activists on the world wide web. Archives of disease in childhood, 87(1), 22-25. doi: http://dx.doi.org/10.1136/adc.87.1.22

Deloitte. (2017). Las vacunas en España. Situación actual y perspectivas de futuro. Recuperado el 11 de febrero de 2020 de https://www2.deloitte.com/content/dam/Deloitte/es/Documents/sanidad/Deloitte-ESsanidad-estudio-vacunas-2017.pdf

Dube, E., Vivion, M., \& MacDonald, N. E. (2015). Vaccine hesitancy, vaccine refusal and the anti-vaccine movement: influence, impact and implications. Expert review of vaccines, 14(1), 99-117.

Ehreth, J. (2003). The global value of vaccination. Vaccine, 21(7-8), 596-600.

World Health Organization, UNICEF, World Bank. (2009). Vacunas e Inmunización: Situación Mundial. World Health Organization.

\section{ANEXO}

\subsection{Listado de vídeos de YouTube utilizados en el análisis}


Análisis del contenido publicado en YouTube, Facebook e internet sobre vacunas y anti vacunas

CNN Chile. (2014, Febrero 4). Carmen Gloria Chaigneau: "No somos una agrupación antivacunas exigimos vacunas seguras" [Archivo de video]. Recuperado de https://www.youtube.com/watch?v=rKh6QQpSL08

NTN24. (2015, Junio 30). Movimiento antivacuna cree es imposible contagiarse de enfermedad circulante: médico [Archivo de video]. Recuperado de https://www.youtube.com/watch?v=i6ng1LCvHJM

La Sexta. (2015, Octubre 30). Medio centenar de niños, sin vacunar en la comarca de La Garrotxa [Archivo de video]. Recuperado de https://www.youtube.com/watch?v=mApGXutPbxM

La Sexta. (2015, Octubre 30). Ortrud Lindemann, doctora: "No hay ninguna vacuna que sea segura al $100 \%$ [Archivo de video]. Recuperado de https://www.youtube.com/watch?v=tSx LWaeTJs

La Sexta. (2015, Octubre 30). El perfil de los antivacunas [Archivo de video]. Recuperado de https://www.youtube.com/watch?v=OwJZyAXIVaM

Preguntas incómodas. (2015, Diciembre 1). 5 Preguntas Incómodas sobre el movimiento antivacunas [Archivo de video]. Recuperado de https://www.youtube.com/watch?v=I6XUd3iM8e0

CNN. (2016, Septiembre 9). Andrea Schilling: "Estos grupos antivacunas son asesinos en potencia" [Archivo de video]. Recuperado de https://www.youtube.com/watch?v=fDRHIuRcTw0

Víctor Hugo Morales. (2017, Julio 2). La campaña anti-vacunas del gobierno [Archivo de video]. Recuperado de https://www.youtube.com/watch?v=ObjObplSR60

Excélsior TV. (2018, Marzo 26). Punto y coma: Movimiento antivacunas [Archivo de video]. Recuperado de https://www.youtube.com/watch?v=gQcIIP8UrE8

Desayunos informales. (2018, Marzo 29). El auge de los movimientos antivacunas y el resurgimiento de enfermedades [Archivo de video]. Recuperado de https://www.youtube.com/watch?v=kxLS4IILk3c

El Siete TV. (2018, Julio 26). Padres antivacunas: Habrá multas de hasta 7600 y arrestos [Archivo de video]. Recuperado de https://www.youtube.com/watch?v=qxXIsKKvObw

Universidad Nacional de Colombia - UN Televisión. (2018, Agosto 9). El movimiento antivacuna ¿una propuesta real o solo una moda? [Archivo de video]. Recuperado de https://www.youtube.com/watch?v=SQ2R6GvalCw 
Análisis del contenido publicado en YouTube, Facebook e internet sobre vacunas y anti vacunas

Meganoticias. (2018, Agosto 17). Ahora Reportajes: Los nocivos efectos de los antivacunas [Archivo de video]. Recuperado de https://www.youtube.com/watch?v=TnhYRGcAONc

Telemiño Ourense. (2018, Agosto 22). Antivacunas y brote de sarampión [Archivo de video]. Recuperado

de https://www.youtube.com/watch?v=k569din7QbY\&feature=emb logo

Reporte Indigo. (2018, Septiembre 21). Los antivacunas, el movimiento que está matando | Reporte Indigo 1586 [Archivo de video]. Recuperado de https://www.youtube.com/watch?v=mi3MYB72pl0

Mauricio Schwarz. (2018, Octubre 1). Mal que les pese a los antivacunas, la vacuna del VPH es segura, efectiva y recomendable [Archivo de video]. Recuperado de https://www.youtube.com/watch?v=3dM3Os7uvz8

La gata de Schrödinger. (2018, Noviembre 1). MOLINOS en el Mar y ANTIVACUNAS en Berlín | Vlog en Alemania [Archivo de video]. Recuperado de https://www.youtube.com/watch?v=w4IMSdcbsBo

Píldoras de psicología. (2018, Diciembre 9). En la mente de los antivacunas: ¿por qué piensan así? [Archivo de video] Recuperado de

\section{https://www.youtube.com/watch?v= iUEBNLDSdg}

Ibarba. (2018, Diciembre 19). ANTIVACUNAS contagian a pequeño bebé DE SARAMPIÓN [Archivo de video]. Recuperado de https://www.youtube.com/watch?v=YelmBhZ9vzM

Danna Alquati. (2019, Enero 12). La gran ESTAFA de los ANTIVACUNAS [Archivo de video]. Recuperado de https://www.youtube.com/watch?v=4gcQPeYkPN4

La nación. (2019, Enero 17). Los anti vacunas, una amenaza según la OMS - Café de la tarde [Archivo de video]. Recuperado de https://www.youtube.com/watch?v=tAk2iBJEVps

T13. (2019, Febrero 12). Hijos de padres antivacuna piden ayuda en redes sociales para vacunarse [Archivo de video]. Recuperado de https://www.youtube.com/watch?v=Q-VXgYVT450

Dr. Andy. (2019, Febrero 22). SOY ANTIVACUNAS// 5 RAZONES para NO VACUNAR A LOS HIJOS [Archivo de video]. Recuperado de https://www.youtube.com/watch?v=leZXk3Qdp20 
Análisis del contenido publicado en YouTube, Facebook e internet sobre vacunas y anti vacunas

Noticias Telemundo. (2019, Marzo 3). Brote de sarampión aviva el debate entre los antivacunas y la ciencia | Noticias Telemundo [Archivo de video]. Recuperado de https://www.youtube.com/watch?v=U5QT7GaBBPw

RT en español. (2019, Marzo 14). ¿Eres más de vacunas o de antivacunas? Aquí está lo que necesitas saber [Archivo de video]. Recuperado de https://www.youtube.com/watch?v=w5U2Vxpr1EM

Tareasplus [Profesor Caliche]. (2019, Marzo 16). EI MOVIMIENTO ANTIVACUNAS: Una MENTIRA que amenaza la SALUD de todos [Archivo de video]. Recuperado de https://www.youtube.com/watch?reload=9\&v=RzplaZxrfHQ

Luisjefe1Vlogs. (2019, Marzo 17). Doctor Antivacunas - Luisjefe1Vlogs [Archivo de video]. Recuperado de https://www.youtube.com/watch?v=BZSca4a6S30

Telenoche. (2019, Marzo 18). Los antivacunas no vacunan a sus hijos y los exponen a todas las enfermedades [Archivo de video]. Recuperado de https://www.youtube.com/watch?v=YVi9R6V8-08

Repretel Costa Rica. (2019, Marzo 25). Grupos antivacunas no afectan brotes de sarampión [Archivo de video]. Recuperado de https://www.youtube.com/watch?v=19t2 1fRj1Y

Mundo TKM. (2019, Marzo 26). El PELIGRO del movimiento ANTI VACUNAS [Archivo de video]. Recuperado de https://www.youtube.com/watch?v=v5mGeBOnckw

Euronews (en español). (2019, Marzo 27). El peor brote de sarampión que enfrenta EEUU en décadas [Archivo de video]. Recuperado de https://www.youtube.com/watch?v=0JyvH992Yxs

Canal 4. (2019, Abril 9). Vespertinas - Movimientos antivacunas ¿por qué no vacunarse? [Archivo de video]. Recuperado de https://www.youtube.com/watch?v=hxEIZjWNGaw

El Trece. (2019, Abril 12). Alerta padres: vacunas si, vacunas no [Archivo de video]. Recuperado de https://www.youtube.com/watch?v=HsGzFIVDuQw

El Siete TV. (2019, Abril 15). El Gobierno rechaza el movimiento antivacunas [Archivo de video]. Recuperado de https://www.youtube.com/watch?v=r y6GVWDK08

Guille Aquino. (2019, Abril 20). Sketch - FAMILIA ANTI VACUNAS [Archivo de video]. Recuperado de https://www.youtube.com/watch?v=i3nRgFKFhFU

C5N. (2019, Abril 25). Padres antivacunas: una tendencia peligrosa que crece [Archivo de video]. Recuperado de https://www.youtube.com/watch?v=BPlidwHI290 
Análisis del contenido publicado en YouTube, Facebook e internet sobre vacunas y anti vacunas

HBO en Latinoamérica. (2019, Mayo 11). \#CHUMELxHBO | Antivacunas [Archivo de video]. Recuperado de https://www.youtube.com/watch?v=UVxKk9ehjFI

Makakiux. (2019, Mayo 14). ANTIVACUNAS EPIDEMIA DE ESTUPIDEZ [Archivo de video]. Recuperado de https://www.youtube.com/watch?v=wJooLuovAWk

Mr Doctor Oficial. (2019, Mayo 19). 5 RAZONES PARA NO VACUNAR A TUS HIJOS ॥ Antivacunas $\|$ MrDoctor [Archivo de video]. Recuperado de https://www.youtube.com/watch?v=xRyHts-TWaQ

Telemundo. (2019, Mayo 21). Mitos del movimiento antivacunas | Noticias Telemundo [Archivo de video]. Recuperado de https://www.youtube.com/watch?v=Ug15UNiCQI0

Marito Baracus. (2019, Junio 4). Rex Anti Vacunas [Archivo de video]. Recuperado de https://www.youtube.com/watch?v=X03Nmgvnh38\&list=LLkq3sAdjPf5ILW5tI2BdRDg \&index $=939$

RFI Español. (2019, Junio 14). \#EnPrimeraPlana 1/2 El auge de los movimientos antivacunas [Archivo de video]. Recuperado de https://www.youtube.com/watch?v=1x9 xzUheNg

RFI Español. (2019, Junio 14). \#EnPrimeraPlana 2/2 El auge de los movimientos antivacunas [Archivo de video]. Recuperado de https://www.youtube.com/watch?v=WxPJ8yVisE8

Plumas Atómicas. (2019, Agosto 31). Sarampión en México, ¿por antivacunas europeos? [Archivo de video]. Recuperado de https://www.youtube.com/watch?v=d2LgKTNTNdl

ADN40. (2019, Septiembre 18). Bladn40 - Antivacunas: ¿una puerta falsa? [Archivo de video]. Recuperado de https://www.youtube.com/watch?v=cTgPOiXMQhE

Mentiras Verdaderas La Red. (2019, Octubre 9). Argentina: 5 niños de padres "antivacunas" se contagiaron con sarampión [Archivo de video]. Recuperado de https://www.youtube.com/watch?v=NYB1 ECuTnc

France 24 en español. (2019, Noviembre 22). Los movimientos antivacunas en una Francia cada vez más desconfiada [Archivo de video]. Recuperado de https://www.youtube.com/watch?v=cC LMjniil8

Afpes. (2019, Diciembre 6). Samoa lucha contra la epidemia de sarampión y los antivacunas I AFP [Archivo de video]. Recuperado de https://www.youtube.com/watch?v=zJO1Ltvo2rl 
Análisis del contenido publicado en YouTube, Facebook e internet sobre vacunas y anti vacunas

\subsection{Listado de blogs}

Roig, V. (2020). El blog de pills [Blog]. Recuperado de https://www.elblogdepills.com/

Isalud [Blog]. Recuperado de https://www.isalud.com/blog/

Live Med Iberia [Blog]. Recuperado de https://www.livemed.in/blog

Jara, M. (2020). Miguel Jara [Blog]. Recuperado de http://www.migueljara.com/

Real e ilustre Colegio de farmacéuticos de Sevilla [Blog]. Recuperado de http://www.farmaceuticosdesevilla.es/blog/

\subsection{Listado de grupos de Facebook}

¡La importancia de las Vacunas! [Nombre de usuario] Recuperado de https://www.facebook.com/groups/541904929740761/

Afectados por el mercurio [Nombre de usuario] Recuperado de https://www.facebook.com/groups/604058640089804/

Anti Antivacunas [Nombre de usuario] Recuperado de https://www.facebook.com/groups/307166246860080/about/

Científicos provacunas [Nombre de usuario] Recuperado de https://www.facebook.com/groups/1232552076771162/about/

El peligro del mercurio en las vacunas [Nombre de usuario] Recuperado de https://www.facebook.com/groups/nomasmercurio/

No vacunas forzadas - No SB277 [Nombre de usuario] Recuperado de https://www.facebook.com/groups/novacunasforzadas/

STOP VACUNAS [Nombre de usuario] Recuperado de https://www.facebook.com/groups/STOPVACUNAS/

\section{AUTORES}

Paloma Piqueiras Conlledo.

Doctora con Mención Internacional en Comunicación Audiovisual, Publicidad y Relaciones Públicas, Máster en Comunicación de las Organizaciones y Licenciada en Periodismo por la Universidad Complutense de Madrid (UCM). En la actualidad, es investigadora postdoctoral y docente en la UCM. Miembro del Grupo de Investigación 
"Intangibles en el Sector Público" y del Comité Académico de la Asociación de Comunicación Política (ACOP). Anteriormente, ha trabajado en la Cadena COPE y en el Departamento de Comunicación de Siemens Healthineers España.

Orcid ID: https://orcid.org/0000-0002-1485-8812

\section{Giorgio De Marchis Picciol.}

Titulado en Ciencias de la Información, Psicología, y Ciencias Económicas y Empresariales y Profesor Contratado Doctor en la Universidad Complutense de Madrid. Sus estudios actuales se centran principalmente en mecanismos psicológicos relacionados con la memoria, las marcas, y los efectos de la mentoría en las organizaciones. Ha publicado en revistas de prestigio como Computers in Human Behavior, Cognitive Processing, o Measuring Business Excellence. Es miembro del Grupo de Investigación "Sentiment y popularidad de los mensajes pro y anti-vacunas en redes: análisis de respuestas explícitas e implícitas mediante EEG, GSR, reconocimiento facial y eye-tracking". Antes de incorporarse al mundo académico ha trabajado en el sector de la comunicación, especialmente publicidad y cine. Es fundador de la Agencia de Comunicación Junior El Estudio de la UCM de la que ha sido Delegado del Decano hasta hace poco.

Orcid ID: https://orcid.org/0000-0001-9217-3141

\section{Victoria Cuesta Díaz}

Doctora en enfermería por la Universidad Complutense de Madrid con sobresaliente cum laude, en la actualidad es enfermera especialista en la UCI de Neonatología del Hospital Quirón Salud de Madrid. Ex profesora del master propio de Comunicación y Salud de la Universidad Complutense. Profesora-tutora de alumnos de Enfermería en prácticas hospitalarias de la Universidad Francisco de Vitoria en el Hospital Quirón Salud de Madrid en el área crítica neonatal. Autora de diferentes artículos y capítulos de libro sobre comunicación y salud.

Orcid ID: https://orcid.org/0000-0003-1318-1173 